\title{
Angle-stable polyaxial locked plating with and without polymethylmethacrylate cement augmentation for proximal humeral fractures in elderly
}

\author{
Sheer Ahmad Hakimi, MD ${ }^{1,2}$, Katharina Schumacher, $\mathrm{MBChB}^{3}\left(\mathbb{D}\right.$, Andrej Ring, $\mathrm{MD}^{2,3}$ (D) \\ 'Department of Orthopedic and Trauma Surgery, St. Josefs Hospital, Dortmund, Germany \\ ${ }^{2}$ Ruhr University, Faculty of Medicine, Bochum, Germany \\ ${ }^{3}$ Department of Reconstructive Surgery, St. Rochus Hospital, Castrop-Rauxel, Germany
}

The proximal humeral fracture (PHF) represents a relatively common osteoporotic fracture type in elderly causing significant morbidity. Around two-thirds of PHFs have been shown to occur in patients aged 60 years and over. ${ }^{[1,2]}$ Numerous surgical techniques for the treatment of displaced, comminuted, or angulated PHFs such as percutaneous techniques, intramedullary nailing, plating, or arthroplasty have been established. ${ }^{[3,4]}$ Although there are advancements in angle-stable polyaxial locked plating (PLP) systems, the outcomes have still not reached desirable levels. ${ }^{[5-7]}$ Brunner et al. ${ }^{[8]}$ reported in their multi-center, prospective study, that mechanical failure requiring revision was seen in $13.8 \%$ of cases. Polyaxial locking plate osteosynthesis remains a challenging procedure with a complication rate of up to $23 \%$ as reported by

Received: July 31, 2021

Accepted: September 30, 2021

Published online: November 19, 2021

Correspondence: Andrej Ring, MD. St. Rochus Hospital, Glückaufstraße 10, 44575 Castrop-Rauxel, Germany.

E-mail: andrej.ring@ruhr-uni-bochum.de

Doi: $10.52312 /$ jdrs.2021.360

Citation: Hakimi SA, Schumacher K, Ring A. Angle-stable polyaxial locked plating with and without polymethylmethacrylate cement augmentation for proximal humeral fractures in elderly. Jt Dis Relat Surg 2021;32(3):575-582.

(O2021 All right reserved by the Turkish Joint Diseases Foundation

This is an open access article under the terms of the Creative Commons Attribution-NonCommercial License, which permits use, distribution and reproduction in any medium, provided the original work is properly cited and is not used for commercial purposes (http://creativecommons.org/licenses/by-nc/4.0/).

\section{ABSTRACT}

Objectives: This study aims to evaluate the outcomes of proximal humeral fracture (PHF) fixation with a polyaxial locking plate (PLP) osteosynthesis alone versus cement-augmented PLP (PLP-CA) in an elderly population.

Patients and methods: Between May 2015 and June 2018, a total of 101 patients (17 males, 84 females; mean age: $74.5 \pm 8.1$ years; range, 60 to 94 years) aged $\geq 60$ years with an acute PHF who underwent osteosynthesis with PLP or PLP-CA were retrospectively analyzed. The patients were divided into two groups as the PLP $(n=53)$ and PLP-CA $(n=48)$. Clinical outcomes, Constant-Murley Scores (CMS), Disabilities of the Arm, Shoulder and Hand (DASH) scores, and Short Form-12 (SF-12) scores were compared between the groups.

Results: The overall mean follow-up was $28.1 \pm 11.1$ months. No clinically relevant differences in the mean duration of surgery, mean intraoperative X-ray image intensifier time or postoperative in-hospital stay were found between the groups. A higher complication rate was observed in the PLP group (20.8\% in PLP vs. $10.4 \%$ in PLP-CA; $\mathrm{p}<0.05$ ). There was no statistically significant difference for this (t-test, $\mathrm{p}=0.08848$ ). The CMS for the operated side did not show any significant differences between the groups. Also, no statically significant difference was seen in the SF-12. A slightly improved DASH score was found for the PLP group ( $\mathrm{p}=0.02908)$.

Conclusion: During follow-up PLP-CA osteosynthesis yielded nearly similar functional outcomes to PLP fracture fixation, despite with an overall lower rate of complication regarding secondary loss of reduction and screw cut-out. The polymethylmethacrylate cement augmentation can decrease morbidity in this patient group.

Keywords: Bone cement, geriatrics, osteoporosis, polymethylmethacrylate.

Königshausen et al. ${ }^{[9]}$ A common problem encountered with this technique is fragment displacement with screw cut-out. In addition, osteoporosis and patient age are usually considered the main risk factors 
for failure of PHF fixation by plating. ${ }^{[8-11]}$ A high complication rate leads to even more morbidity and need for revision surgery.

Augmenting fragility fractures has a potential to improve orthopedic interventions to the porotic bone. The polymethylmethacrylate (PMMA) cement augmentation has been shown to be effective in biomechanical studies. Röderer et al. ${ }^{[12]}$ investigated the outcome of cement augmentation in locking plate osteosynthesis in a PHF model. The researchers used human humerus specimens in which three-part fractures with a metaphyseal defect were simulated and fixed with the plate. For augmentation, bone cement was used and a cyclic varus bending test with increasing upper load magnitude was performed, until failure of the screw-bone fixation occurred. The results demonstrated that the cement augmentation within the humeral head in locked plating was effective in improving primary stability. The augmented group withstood significantly more load cycles in a cyclic varus-bending test. The role of cement augmentation in pathological fractures of the extremity is well documented; it is thought to prevent implant failure and increase stability. ${ }^{[13-15]}$ The PMMA is still a valid alternative for augmentation with its low cost, although ceramic bone substitutes may be potentially more effective. There is undoubtedly need for clinical studies to establish the role of augmentation as a routine practice.

Although the research on this topic is limited, the multi-center, randomized-controlled trial by Hengg et al. ${ }^{[16]}$ compared the outcome of locking plate augmentation with and without PMMA augmentation. However, their study was prematurely terminated after 67 patients were enrolled, and the outcomes did not reach statistical power to detect differences.

In the present study, we hypothesized that cementaugmented PLP (PLP-CA) osteosynthesis could provide at least equivalent clinical outcomes, with less secondary fracture displacement, compared to PLP alone. We, therefore, aimed to evaluate the outcomes of PHF fixation with a PLP alone versus PLP-CA in an elderly population.

\section{PATIENTS AND METHODS}

This single-center, retrospective, observational study was conducted at St. Josefs Hospital Dortmund, Department of Orthopedic and Trauma Surgery between May 2015 and June 2018. A total of 211 patients aged 60 or above with an acute isolated PHF were screened. Of these patients, 101 (17 males, 84 females; mean age: $74.5 \pm 8.1$ years; range, 60 to 94 years) met the inclusion criteria and were included in the study. Inclusion criteria were as follows: age $\geq 60$ years, having surgical treatment of the fracture by polyaxial plate osteosynthesis. Patients who had prior surgical treatment of the proximal humerus or neurological damage at time of presentation and those having four-part fractures were excluded. Also, patients who already died at the time of the evaluation $(n=67)$, who suffered from dementia $(\mathrm{n}=20)$, and who by choice or as a result of physical impairment due to other illnesses could not or were unwilling to participate in the study $(n=23)$ were excluded. A follow-up of at least nine months was defined as a further inclusion criterion. A written informed consent was obtained from each patient. The study protocol was approved by the Medical Board Westfalen-Lippe Ethics Committee (2018-391-f-S). The study was conducted in accordance with the principles of the Declaration of Helsinki.

All patients were categorized into two groups: PLP $(n=53)$ and PLP-CA $\quad(n=48)$. Fractures were classified according to the Neer classification. ${ }^{[17]}$ Data including patient demographics, clinical characteristics, dominance of shoulder and American Society of Anesthesiologists (ASA) class, and operative data were recorded.

In our institution, a simple algorithm for the osteosynthetic treatment of PHFs has been developed in recent years. In otherwise healthy patients under 60 years of age, plates are usually used alone. The decision to use cement augmentation is an option for patients over 60 years of age with known osteoporosis and reduced bone density; i.e., cortical thickness on radiographs. The decisive factor, however, is the surgeon's experience and assessment of the intraoperative findings (bone quality). In addition to bone quality, comorbidities such as heart failure and usually a higher-grade of mobility impairment, but also the patient's gait unsteadiness and the tendency to fall, play a role in the decision for cement augmentation.

In our institution, the standard protocol is as follows: surgery is performed in the beach-chair position and under general anesthesia. Angle-stable, titanium PLP system (Königsee Implantate, Allendorf, Germany) is used via deltopectoral approach. Polymethylmethacrylate is used for cement augmentation (Palacos ${ }^{\circledR}$, Heraeus Medical, Hanau, Germany) based on the opinion of the experienced surgeon. ${ }^{[18]}$ 


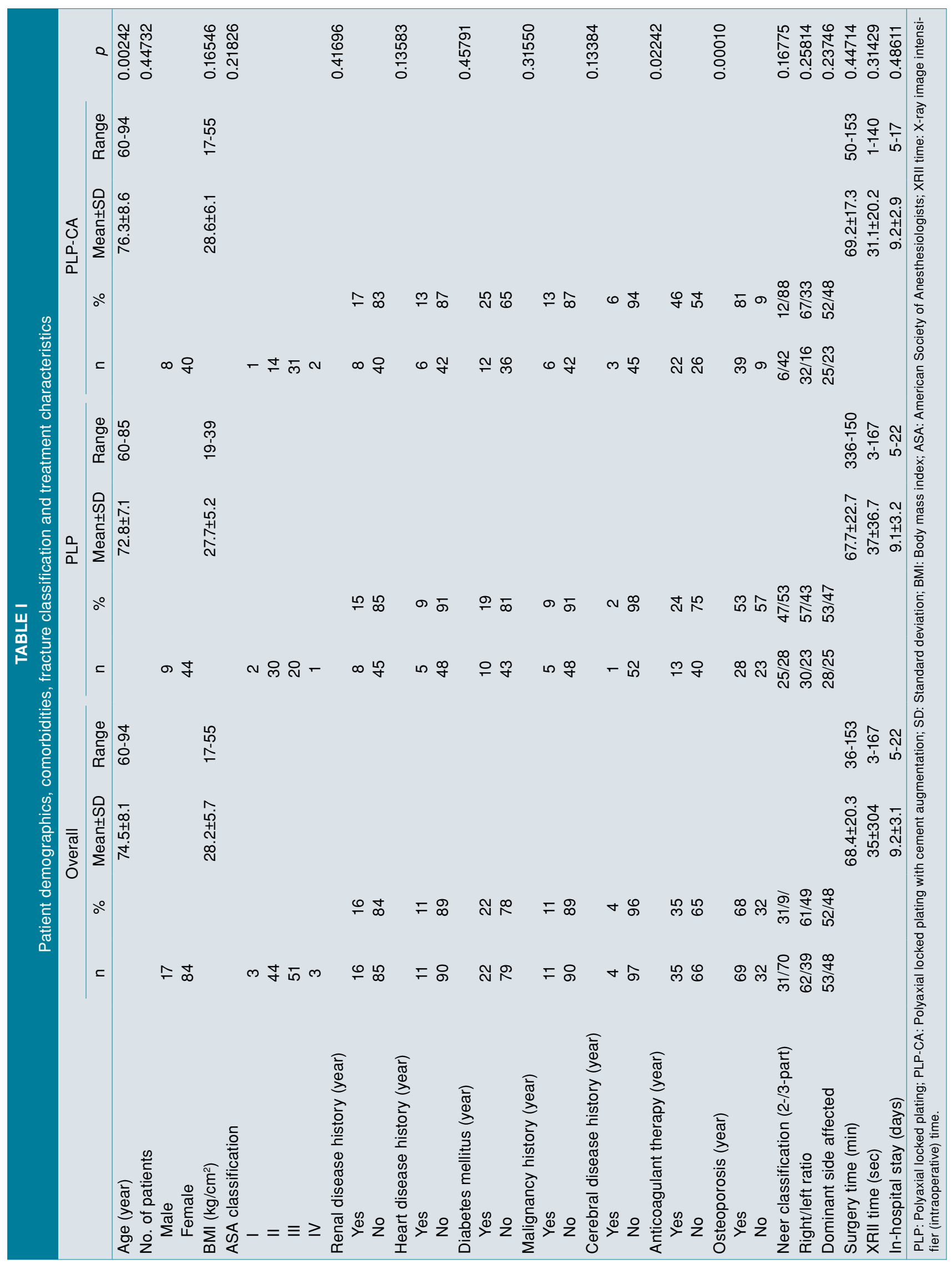


In this study, the preparation of the PMMA cement was performed according to the instruction guide. The implantation of the cement in metaphysical defect zone was done after the reduction of the fracture. The reduction result was controlled under X-ray image intensifier, before the implant was used.

In both groups, postoperative protocol was the same, including active and passive exercises of the shoulder initiated on the day following surgery. The patients were instructed not to loadbear with the affected shoulder for six weeks postoperatively. In the follow-up evaluation, the shoulder function was assessed by an independent specialist using the Constant-Murley Score (CMS) at least nine months after operation. ${ }^{[19]}$ Further outcome measure included Disabilities of the Arm, Shoulder and Hand (DASH) score. ${ }^{[20]}$ The 12-item Short Form-12 (SF-12) was used to evaluate the patient-reported impact of health on an individual's everyday life. ${ }^{[21]}$

\section{Statistical analysis}

Statistical analysis was performed using the IBM SPSS version 25.0 software (IBM Corp., Armonk, NY, USA). Descriptive data were expressed in mean \pm standard deviation (SD), median (min-max) or number and frequency, where applicable. The
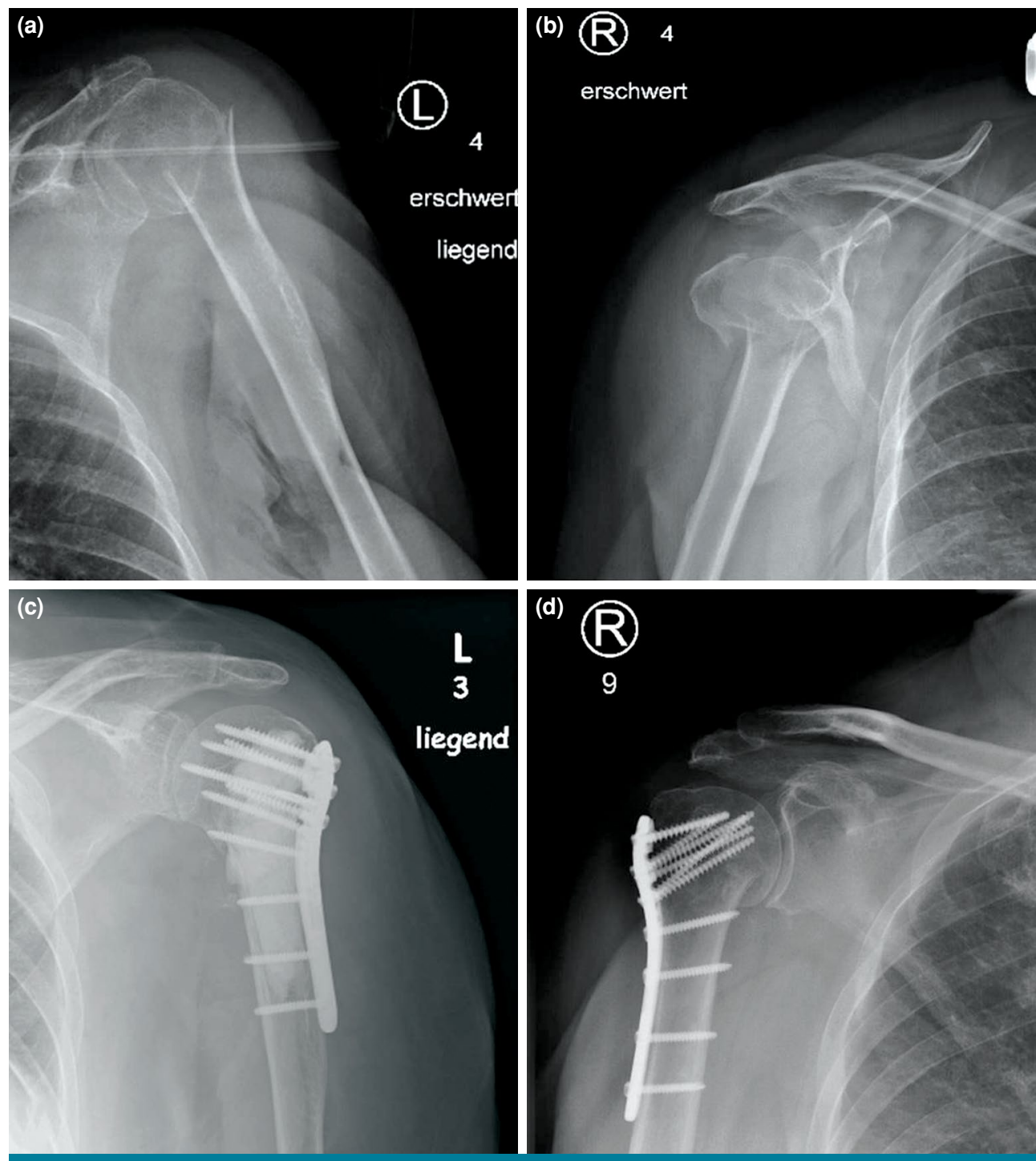

FIGURE 1. Radiographic images of the injured shoulders of two female patients, 88 (a) and 70 (b) years old, before and eight weeks after surgical fracture treatment by angle-stable polyaxial locked plating with (c) and without (d) the use of PMMA cement.

PMMA: Polymethylmethacrylate; L: Left; R: Right; "liegend"=supine; "erschwert"=difficult imaging. 
Kolmogorov-Smirnov-test was used to test for normal distribution. In case of normal distribution, an unpaired t-test was used. A $p$ value of $<0.05$ was considered statistically significant.

\section{RESULTS}

Of a total of 101 patients included in the study, there were 53 patents in the PLP group and 48 patients in the PLP-CA group. The overall mean follow-up was $28.1 \pm 11.1$ (range, 9 to 64 ) months. The majority of patients had three-part fractures, corresponding to 70 patients $(69 \%)$ in both groups according to the Neer classification. The PLP group consisted of $53 \%$ of three-part fractures, whereas $88 \%$ in the PLP-CA group were three-part fractures. There were no significant differences in baseline characteristics between the two groups (Table I).

Osteoporosis diagnosed prior to PHF existed in $81 \%$ of the PLP-CA and in $53 \%$ of the PLP groups $(p=0.00010)$. However, the previously known osteoporosis was already treated with medication in only $17 \%$ of the PLP group and in $38 \%$ of the PLP-CA group. Regarding the mean age, a significant difference was seen $(72.8 \pm 7.1$ years in PLP vs. $76.3 \pm 8.6$ years in PLP-CA; $p=0.00242$ ) (Table I).

The mean duration of surgery showed no statistically significant difference between the groups (67.7 $\pm 22.7 \mathrm{~min}$ in PLP and $69.2 \pm 17.3 \mathrm{~min}$ in PLP-CA; $\mathrm{p}>0.05)$. Satisfactory fracture reduction was achieved in all cases (Figure 1a-d). The mean length of hospital stay did not differ significantly between the PLP and PLP-CA groups (Table I).

No significant differences were seen between the PLP and PLP-CA groups according to the CMS scores (Table II). In particular, the shoulder range of flexion and abduction revealed no significant difference between the groups. The PLP group showed a slightly reduced mean DASH scores compared to the PLP-CA group (26.4 \pm 23.2 vs. $32.5 \pm 23.2$, respectively; $\mathrm{p}=0.02908$ ). However, the physical and mental evaluation of the SF-12 questionnaire with overall improved values for PLP group revealed no statistically significant differences (Table II).

A total of 11 complications (20.8\%) in PLP group and five complications $(10.4 \%)$ in PLP-CA group were recorded $(p=0.08848)$. The most frequent complication was a secondary displacement of the humeral head with screw cut-out, which occurred in four patients in the PLP group and one patient in the PLP-CA group. A secondary failure of fixation, without screw cut-out, was noted in three patients in the PLP group. The second most frequent complication was a persistent pain, which was seen in two patients in the PLP and in one patient in the PLP-CA group (Table III).

Further complications were avascular head osteonecrosis in one patient in the PLP group and in another patient in the PLP-CA group. One patient in the PLP and one patient in the PLP-CA groups suffered from a postoperative infection.

Overall, further surgery was indicated in 12 patients (PLP, $n=10$ and PLP-CA, $n=2$ ) due to the associated complications. All operations were performed during the follow-up period, with implant removal and arthrolysis being the most frequent secondary procedure. In two patients, a revision to total shoulder joint replacement was done. No fracture complexity compared two-part with three-part fractures was seen to have any

\begin{tabular}{|c|c|c|c|c|c|c|c|c|c|c|}
\hline \multicolumn{11}{|c|}{$\begin{array}{l}\text { TABLE II } \\
\text { the SF-12 survey }\end{array}$} \\
\hline & \multicolumn{3}{|c|}{ Overall } & \multicolumn{3}{|c|}{ PLP } & \multicolumn{3}{|c|}{ PLP-CA } & \multirow[b]{2}{*}{$p$} \\
\hline & $\mathrm{n}$ & Mean $\pm S D$ & Range & $\mathrm{n}$ & Mean $\pm S D$ & Range & $\mathrm{n}$ & Mean $\pm S D$ & Range & \\
\hline No. of patients & 101 & & & 53 & & & 48 & & & \\
\hline Follow-up (m) & & $28.1 \pm 11.1$ & $9-64$ & & $28.5 \pm 11.4$ & $10-51$ & & $27.5 \pm 10.7$ & $9-64$ & 0.39252 \\
\hline CS OP & & $66 \pm 16.7$ & $27-88$ & & $66.9 \pm 17.5$ & $27-88$ & & $65 \pm 15.8$ & $28-86$ & 0.37957 \\
\hline CS CL & & $79.5 \pm 5.9$ & $58-88$ & & $81 \pm 4.8$ & $68-88$ & & $77.7 \pm 6.6$ & $58-86$ & 0.02559 \\
\hline DASH score & & $31.8 \pm 22.9$ & $0-78.9$ & & $26.4 \pm 23.2$ & $0-74.2$ & & $32.5 \pm 23.2$ & $0-78.9$ & 0.02908 \\
\hline SF-12 PS & & $38.7 \pm 11.2$ & $15-57$ & & $39.7 \pm 11.7$ & $17-57$ & & $37.7 \pm 10.6$ & $15-56$ & 0.29938 \\
\hline SF-12 MS & & $52 \pm 11.1$ & $23-70$ & & $52.7 \pm 11.6$ & $23-70$ & & $51.2 \pm 10.5$ & $26-65$ & 0.25950 \\
\hline
\end{tabular}




\begin{tabular}{|c|c|c|c|c|c|c|c|}
\hline & & l comp & & tients & & & \\
\hline & & & & & & & \\
\hline & $\mathrm{n}$ & $\%$ & $\mathrm{n}$ & $\%$ & $\mathrm{n}$ & $\%$ & $p$ \\
\hline No. of patients & 101 & & 53 & & 48 & & \\
\hline Complications & 16 & 15.8 & 11 & 20.8 & 5 & 10.4 & \\
\hline Cut-out & 5 & 31 & 4 & 36 & 1 & 20 & \\
\hline Secondary displacement & 4 & 25 & 3 & 27 & 1 & 20 & 0.08848 \\
\hline Avascular osteonecrosis & 2 & 12 & 1 & 9 & 1 & 20 & \\
\hline Wound infection & 2 & 12 & 1 & 9 & 1 & 20 & \\
\hline Persistent pain & 3 & 19 & 2 & 18 & 1 & 20 & \\
\hline
\end{tabular}

correlation with overall complications in either the PLP or PLP-CA groups.

\section{DISCUSSION}

In the study, we present the outcomes of PLP osteosynthesis compared to locked plating using PMMA cement augmentation. During follow-up, PLP-CA osteosynthesis reached similar functional outcomes to PLP fracture fixation, despite with a lower rate of complication regarding secondary loss of reduction and screw cut-out. A higher rate of secondary loss of reduction with concomitant screw penetration was seen in the PLP group. The complications in the PLP, particularly the loss of reduction, occurred three times more frequently than in the cement augmented group. With a comparable average age of overall 74 years, a mean CMS score of $66 \pm 16.7$, a DASH score of less than $31.8 \pm 22.9$, as well as a SF-12 physical score above $38.7 \pm 11.2$, both groups in the present study demonstrated positive clinical outcomes at the mean follow-up of $28.1 \pm 11.1$ months. Except for the slightly improved, but clinically insignificant difference in the DASH score for the PLP group, no statistically significant differences in the functional outcomes for the CMS scores was observed between both groups. Likewise, no significant difference in the mean intraoperative $X$-ray image intensifier time or postoperative in-hospital stay was seen between the two groups.

It is well known that the extreme lack of bone mass in the humerus makes fracture synthesis difficult. Therefore, many studies question the choice of suitable osteosynthetic procedures. Surgical techniques for treating osteoporotic PHFs continue to develop, as the ideal treatment strategy has yet to be established. Various biomechanical and clinical investigations have been performed to achieve stable implant anchorage in the weak bone, even while using locking plates. To enhance stability in internal fixation and to avoid implant failure requiring revision surgery, several techniques have been tested (e.g., autografts and allografts, calcium phosphate and calcium sulfate cements). ${ }^{[22-26]}$ Calcium phosphate cement as a potential alternative to implant augmentation in the treatment of osteoporotic humeral head fractures have been also investigated and proved clinically. Kwon et al. ${ }^{[27]}$ conducted a biomechanical evaluation with calcium phosphate cement in cadaveric limbs. Supplementation with calcium phosphate cement led to significant improvements in the mechanical performance of the internal fixation, even in most osteoporotic specimens. In a clinical study, Robinson and Page ${ }^{[25]}$ treated severely impacted valgus fractures by internal fixation and augmentation with injectable calcium phosphate in the humeral head. All fractures united within the first year, all reductions were maintained, and no patient had signs of osteonecrosis of the humeral head. The median CMS score was 80 points and the median DASH score was 22 points. Grünewald et al. ${ }^{[28]}$ performed a dynamic biomechanical evaluation of a calcium phosphate cement paste implanted to augment intramedullary nail fixation of humeral head fracture model. The augmented group withstood significantly more cycles of dynamic axial loading before implant failure. In a clinical study by Lee and Shin, ${ }^{[26]}$ a calcium sulfate injection in the metaphysis was performed to maintain the position of the reduction. Compared to the patients who did not receive cement augmentation, the mean functional score was higher than in those who did not receive the graft.

Polymethylmethacrylate was first employed by orthopedic surgeons over 70 years ago, and remains one of the most enduring materials in orthopedic 
surgery. It still has a central role in the success of total joint replacement. ${ }^{[18]}$

A recent study by Hengg et al. ${ }^{[16]}$ also compared the risk of mechanical failure after locking plate treatment of PHF with or without PMMA cement augmentation. Their multi-center, randomized trial enrolled 67 patients aged over 64 years with displaced PHF from eight European centers. No statistically significant differences in function as measured by DASH and CMS scores were observed between the study groups. According to the authors, the major limitation of their study was its premature termination and a much smaller patient population than the originally planned. Thus, the study did not have the statistical power to detect differences between the groups. However, our results are supported by the finding of Katthagen et al. ${ }^{[29]}$ which compared 24 patients treated by locked plating of displaced PHF with additional cement augmentation and showed similar clinical outcomes, but reduced rate of early implant-related complications compared to locked plating without cement augmentation.

There are some limitations to this study. First, it has a retrospective design. Second, a study in an elderly population inevitably has a high drop-out rate due to comorbidities or death of the patients, presenting difficulties for longer term follow-up. Third, with a follow-up of 28 months, long-term complications may be underestimated. However, no previous studies on PHFs in an elderly population have reported clinical outcomes later than 24 months postoperatively. Finally, in this study, there was only follow-up data available for 101 patients, which limits the power of the study. In addition, a potential bias in cementation procedure cannot be excluded.

Likewise, the lack of evaluation of radiographic parameters is a significant limitation of our study. Unfortunately, we did not carry out any evaluation of objective radiographic parameters such as neck-shaft angle during the follow-up. The focus of our study was more on the assessment of the clinical situation after fracture treatment and not on the recording of radiological parameters. In our opinion, a satisfactory reduction, which did not require an immediate revision, could be achieved intraoperative in most of the patients, but we did not measure this on radiographs.

These limitations are, however, compensated by various strengths, including a homogeneous population, strict inclusion and exclusion criteria, and the use of defined implants. Although not observed by the authors, the potential risk of thermal-induced necrosis on surrounding tissues by using PMMA in augmentation procedures must be also pointed out. Besides, cement leakage into the joint has been described in the literature. ${ }^{[28]}$

In conclusion, implant anchoring in highly osteoporotic bone is challenging. The main findings of the current study are that, during follow-up, PLP-CA osteosynthesis can yield nearly similar functional outcomes to PLP fracture fixation, despite with an overall lower rate of complication regarding secondary loss of reduction and screw cut-out. Based on our study results, we, therefore, recommend implementation of PMMA cement augmentation to PLP osteosynthesis as a standard surgical procedure for the osteoporotic PHFs in the elderly. Treatment of osteoporosis should also be initiated at the time of fracture presentation.

\section{Acknowledgements}

Parts of this work will be accredited to the thesis of Mr. Sheer Ahmad Hakimi at the Ruhr University Bochum.

\section{Declaration of conflicting interests}

The authors declared no conflicts of interest with respect to the authorship and/or publication of this article.

\section{Funding}

The authors received no financial support for the research and/or authorship of this article.

\section{REFERENCES}

1. Bergdahl C, Ekholm C, Wennergren D, Nilsson F, Möller M. Epidemiology and patho-anatomical pattern of 2,011 humeral fractures: data from the Swedish Fracture Register. BMC Musculoskelet Disord 2016;17:159.

2. Palvanen M, Kannus P, Niemi S, Parkkari J. Update in the epidemiology of proximal humeral fractures. Clin Orthop Relat Res 2006;442:87-92.

3. Schumaier A, Grawe B. Proximal Humerus Fractures: Evaluation and management in the elderly patient. Geriatr Orthop Surg Rehabil 2018;9:2151458517750516.

4. Lander ST, Mahmood B, Maceroli MA, Byrd J, Elfar JC, Ketz JP, et al. Mortality rates of humerus fractures in the elderly: Does surgical treatment matter? J Orthop Trauma 2019;33:361-5.

5. Sproul RC, Iyengar JJ, Devcic Z, Feeley BT. A systematic review of locking plate fixation of proximal humerus fractures. Injury 2011;42:408-13.

6. Schliemann B, Siemoneit J, Theisen Ch, Kösters C, Weimann A, Raschke MJ. Complex fractures of the proximal humerus in the elderly--outcome and complications after locking plate fixation. Musculoskelet Surg 2012;96 Suppl 1:S3-11.

7. Gupta AK, Harris JD, Erickson BJ, Abrams GD, Bruce B, McCormick F, et al. Surgical management of complex proximal humerus fractures-a systematic review of 92 studies including 4500 patients. J Orthop Trauma 2015;29:54-9.

8. Brunner F, Sommer C, Bahrs C, Heuwinkel R, Hafner C, Rillmann $\mathrm{P}$, et al. Open reduction and internal fixation of 
proximal humerus fractures using a proximal humeral locked plate: A prospective multicenter analysis. J Orthop Trauma 2009;23:163-72.

9. Königshausen M, Kübler L, Godry H, Citak M, Schildhauer TA, Seybold D. Clinical outcome and complications using a polyaxial locking plate in the treatment of displaced proximal humerus fractures. A reliable system? Injury 2012;43:223-31.

10. Jung SW, Shim SB, Kim HM, Lee JH, Lim HS. Factors that influence reduction loss in proximal humerus fracture surgery. J Orthop Trauma 2015;29:276-82.

11. Hardeman F, Bollars P, Donnelly M, Bellemans J, Nijs S. Predictive factors for functional outcome and failure in angular stable osteosynthesis of the proximal humerus. Injury 2012;43:153-8.

12. Röderer G, Scola A, Schmölz W, Gebhard F, Windolf M, Hofmann-Fliri L. Biomechanical in vitro assessment of screw augmentation in locked plating of proximal humerus fractures. Injury 2013;44:1327-32.

13. Siegel HJ, Lopez-Ben R, Mann JP, Ponce BA. Pathological fractures of the proximal humerus treated with a proximal humeral locking plate and bone cement. J Bone Joint Surg Br 2010;92:707-12.

14. Dhaliwal J, Seif A, Singh S, Sinha A. Cement augmentation of bone defect in pathological humeral diaphyseal fracture treated with retrograde intramedullary nail. Ann R Coll Surg Engl 2012;94:444-5.

15. NamdariS, Voleti PB, Mehta S. Evaluation of the osteoporotic proximal humeral fracture and strategies for structural augmentation during surgical treatment. J Shoulder Elbow Surg 2012;21:1787-95.

16. Hengg C, Nijs S, Klopfer T, Jaeger M, Platz A, Pohlemann $\mathrm{T}$, et al. Cement augmentation of the proximal humerus internal locking system in elderly patients: A multicenter randomized controlled trial. Arch Orthop Trauma Surg 2019;139:927-42.

17. Neer CS 2nd. Displaced proximal humeral fractures: Part I. classification and evaluation. 1970. Clin Orthop Relat Res 2006;442:77-82.

18. Webb JC, Spencer RF. The role of polymethylmethacrylate bone cement in modern orthopaedic surgery. J Bone Joint Surg [Br] 2007;89:851-7.

19. Constant CR, Murley AH. A clinical method of functional assessment of the shoulder. Clin Orthop Relat Res 1987;(214):160-4.

20. Gummesson C, Atroshi I, Ekdahl C. The disabilities of the arm, shoulder and hand (DASH) outcome questionnaire: Llongitudinal construct validity and measuring self-rated health change after surgery. BMC Musculoskelet Disord 2003;4:11.

21. Ware J Jr, Kosinski M, Keller SD. A 12-item short-form health survey: Construction of scales and preliminary tests of reliability and validity. Med Care 1996;34:220-33.

22. Weeks CA, Begum F, Beaupre LA, Carey JP, Adeeb S, Bouliane MJ. Locking plate fixation of proximal humeral fractures with impaction of the fracture site to restore medial column support: Aa biomechanical study. J Shoulder Elbow Surg 2013;22:1552-7.

23. Gardner MJ, Boraiah S, Helfet DL, Lorich DG. Indirect medial reduction and strut support of proximal humerus fractures using an endosteal implant. J Orthop Trauma 2008;22:195-200.

24. Neviaser AS, Hettrich CM, Beamer BS, Dines JS, Lorich DG. Endosteal strut augment reduces complications associated with proximal humeral locking plates. Clin Orthop Relat Res 2011;469:3300-6.

25. Robinson CM, Page RS. Severely impacted valgus proximal humeral fractures. Results of operative treatment. J Bone Joint Surg [Am] 2003;85:1647-55.

26. Lee CW, Shin SJ. Prognostic factors for unstable proximal humeral fractures treated with locking-plate fixation. J Shoulder Elbow Surg 2009;18:83-8.

27. Kwon BK, Goertzen DJ, O'Brien PJ, Broekhuyse HM, Oxland TR. Biomechanical evaluation of proximal humeral fracture fixation supplemented with calcium phosphate cement. J Bone Joint Surg Am 2002;84:951-61.

28. Grünewald D, Langenmair E, Hirschmüller A, Maier D, Südkamp NP, Konstantinidis L. Biomechanical in vitro evaluation of a ready-to-use calcium phosphate cement implanted to augment intramedullary nail fixation of a three-part humeral head fracture model. Proc Inst Mech Eng H 2019;233:706-11.

29. Katthagen JC, Lutz O, Voigt C, Lill H, Ellwein A. Cement augmentation of humeral head screws reduces early implant-related complications after locked plating of proximal humeral fractures. Obere Extrem 2018;13:123-9. 